\title{
Bricks, butter, and slices of cucumber: Investigating semantic influences in amodal completion
}

\author{
Sven Vrins, Tessa C J de Wit $\uparrow$, Rob van Lier \\ Donders Institute for Brain, Cognition and Behaviour, Radboud University Nijmegen, PO Box 9104 , \\ 6500 HE Nijmegen, The Netherlands; e-mail: s.vrins@donders.ru.nl; $\uparrow$ Psychiatry Department, \\ Radboud University Nijmegen Medical Centre, PO Box 9101, 6500 HB Nijmegen, The Netherlands \\ Received 19 December 2007, in revised form 17 July 2008; published online 8 January 2009
}

\begin{abstract}
Objects in our world are partly occluded by other objects or sometimes even partly by themselves. Amodal completion is a visual process that enables us to perceive these objects as complete and is influenced by both local object information, present at contour intersections, and overall (global) object shape. In contrast, object semantics have been demonstrated to play no role in amodal completion but do so only by means of subjective methods. In the present study, object semantics were operationalised by material hardness of familiar objects which was varied to test whether it leaves amodal completion unaffected. Specifically, we investigated the perceived form of joined naturalistic objects that differ in perceived material hardness, employing the primed matching paradigm. In experiments 1 and 2, probing three different prime durations, amodal completion of a notched circular object changes systematically with the hardness of the object it was joined to. These results are in line with the view that amodal completion is inseparable from general object interpretation, during which object semantics may dominate.
\end{abstract}

\section{Introduction}

One of the key problems in extrapolating complete object shape from its visible part(s), is that the number of possible object interpretations is (in principle) infinite. For example, when looking at the partly occluded stimulus on the left of figure 1, the two most plausible object interpretations are a full-circle or a pacman shape. In principle, our visual system could have come up with dozens of circle-like alternatives. However, it is unlikely that our visual system has the time or the resources to go about computing all possible perceptual solutions to eventually provide conscious perception with the sense of one specific whole object. Instead, we would expect amodal completion to be constrained. In fact, for the partly occluded stimulus in figure 1, observers have a strong preference for interpreting the left shape as a circle that lies behind a rectangle (eg Sekuler and Palmer 1992).

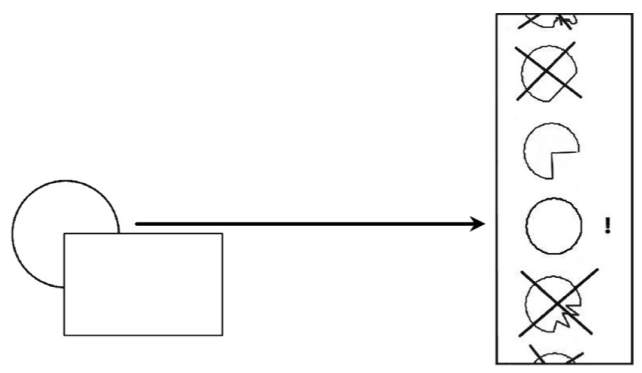

Figure 1. Example of a 2-D occlusion stimulus and (possible) completions.

Several researchers have indeed found distinct rules that govern amodal completion. Their experiments showed that some completions build on local information such as Tand L-junctions of contours of occluding objects (eg Rock 1983; Boselie and Wouterlood 1989; Kellman and Shipley 1991; Rubin 2001; de Wit et al 2008), while others follow from global object characteristics, such as repetition and symmetry (eg van Lier et al 1995; 
de Wit et al 2006). Note that both local and global influences of amodal completion are thus far mainly contour- or texture-driven.

In the past few years, the stimulus domain of mostly 2-D stylistic stimuli has been extended to pictorial 3-D shapes (Tse 1998, 1999, 2002; van Lier and Wagemans 1999) and quasi-regular shapes (van Lier 1999; de Wit and van Lier 2002; de Wit et al 2005). The latter studies demonstrate that the relation between the overall characteristics of a partly occluded object and its preferred shape interpretation does not need to be exact. For example, partly occluded quasi-regular shapes (such as the left shape in figure 2) give rise to a set of similar completions rather than to one specific completion (van Lier 1999; de Wit and van Lier 2002). As can be seen in figure 2, the contour of the partly occluded star-like shape describes a repetition of concave and convex angles, forming its protrusions. Manipulating these angles produced different lengths of protrusions, which affected the overall object regularity. The results showed that participants perceived completions in which the extension of the protrusions fell within a certain range. That is, they were not too large (as in the upper-left completion), too small, or absent (as in the lower-right completion). These findings underpin the view that our visual system is sensitive to global object characteristics, such as shape regularity, even when it is imperfect. Moreover, instead of being tied to one specific completion, the visual system allows a variety of possibilities, based on an object's overall regularity, albeit within a certain range.

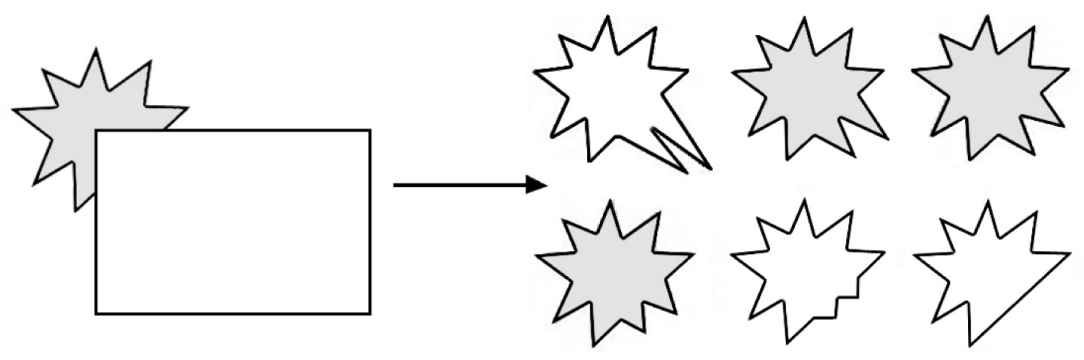

Figure 2. On the left is a quasi-regular occluded shape. The grey shapes on the right are part of the set of perceived plausible completions based on global stimulus properties of the left shape. The white shapes lie outside the set of perceived completions (after de Wit and van Lier 2002).

Gerbino and Zabai (2003) discussed a special case of occlusion, namely object joints. Optically, no shape-information is available on intersection of the object, and the viewer is left with different possible interpretations. Possibly, though this is not very plausible, the joint constitutes parts of both objects or is an empty space. More likely, however, is that one of the two objects is fully convex and the other indented. Indeed, Gerbino and Zabai found several conditions under which the perceived completion switched between completion of one or the other object. Put briefly, objects that are positioned relatively higher and objects that are relatively small are more often amodally completed.

Besides varying such object relations, Gerbino and Zabai discussed the role of object familiarity in completion and argued that it has no influence on the perception of joints. This is in line with earlier work on the relation between perception and knowledge by Kanizsa $(1979,1985)$, who argued that amodal completion follows autonomous principles of organisation, that can lead to percepts that conflict with knowledge-based expectancies. Demonstrating that knowledge does not guide perception, Gerbino and Zabai asked viewers to indicate how they perceived object joints of familiar objects such as an indented banana adjoining a brick (figure 3). Gerbino and Zabai hypothesised that, if common sense about the hardness of the materials constituting the objects affects amodal completion, observers would report seeing an indented banana. Conversely, if completion results from mere metrical or positional 


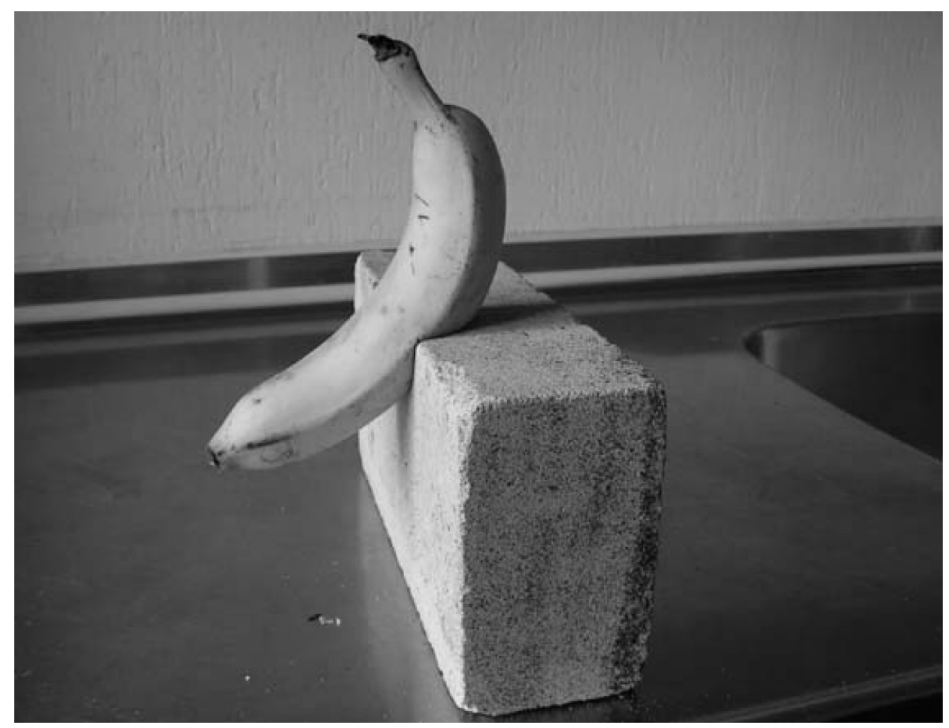

Figure 3. An ambiguous joint of compound objects (after Gerbino and Zabai 2003).

qualities of the stimulus (eg relative sizes and positions along the vertical), observers would report perceiving the banana as complete. Most observers stated that they perceived a whole banana, despite the fact that the different hardnesses of the material implied otherwise. Although Gerbino and Zabai suggested that visual phenomenology is insensitive to knowledge about the material hardness of the objects, they did not dismiss a possible role this could play during the process of amodal completion. Indeed, explicitly retrieving an observer's percept of the indented banana abutting a brick, does not rule out such an influence.

The goal of the present study was to investigate whether these influences can in fact occur during the microgenesis of amodal completion. Instead of letting participants reason subjectively about object configurations, the primed-matching paradigm (Sekuler and Palmer 1992; Sekuler 1994; van Lier et al 1995; Bruno et al 1997; de Wit and van Lier 2002; de Wit et al 2005) was used, which indirectly taps into the participants' percept. Similar to a simple matching task, participants have to indicate as quickly as possible whether two presented shapes (forming a test-pair) are identical (matching) or not. When a matching test-pair is preceded by a shape (a prime) for a certain duration (prime duration), reaction times (RTs) change systematically. If the prime is similar to the test-pair, the RT decreases, which is called a priming effect. What is more, it is the similarity between the prime percept and the test-pair that actually determines the priming effect. For instance, a partly occluded circle, such as in figure 1, has a priming effect on test-pairs of full circles and not on those of pacman shapes. In this way, the primedmatching paradigm can indirectly expose the percept of an ambiguous occlusion prime (relative to prime duration). We used this paradigm to reveal the observers' percept of joined naturalistic shapes. For instance, the cucumber slice in the cucumber-and-brick stimulus (top-left in figure 4) can be interpreted as a full circular slice or a notched slice (pacman). If presenting this cucumber-and-brick prime reduces RTs to test-pairs of full cucumber-slices, the joint is perceived as a cucumber penetrating the brick. Conversely, if RTs to test-pairs of notched cucumber-slices are reduced, the joint is perceived as a brick penetrating the cucumber. In the first case, the results would provide no evidence of any effect related to familiarity with the material hardness of the objects (ie object semantics). In the latter case, they would. 

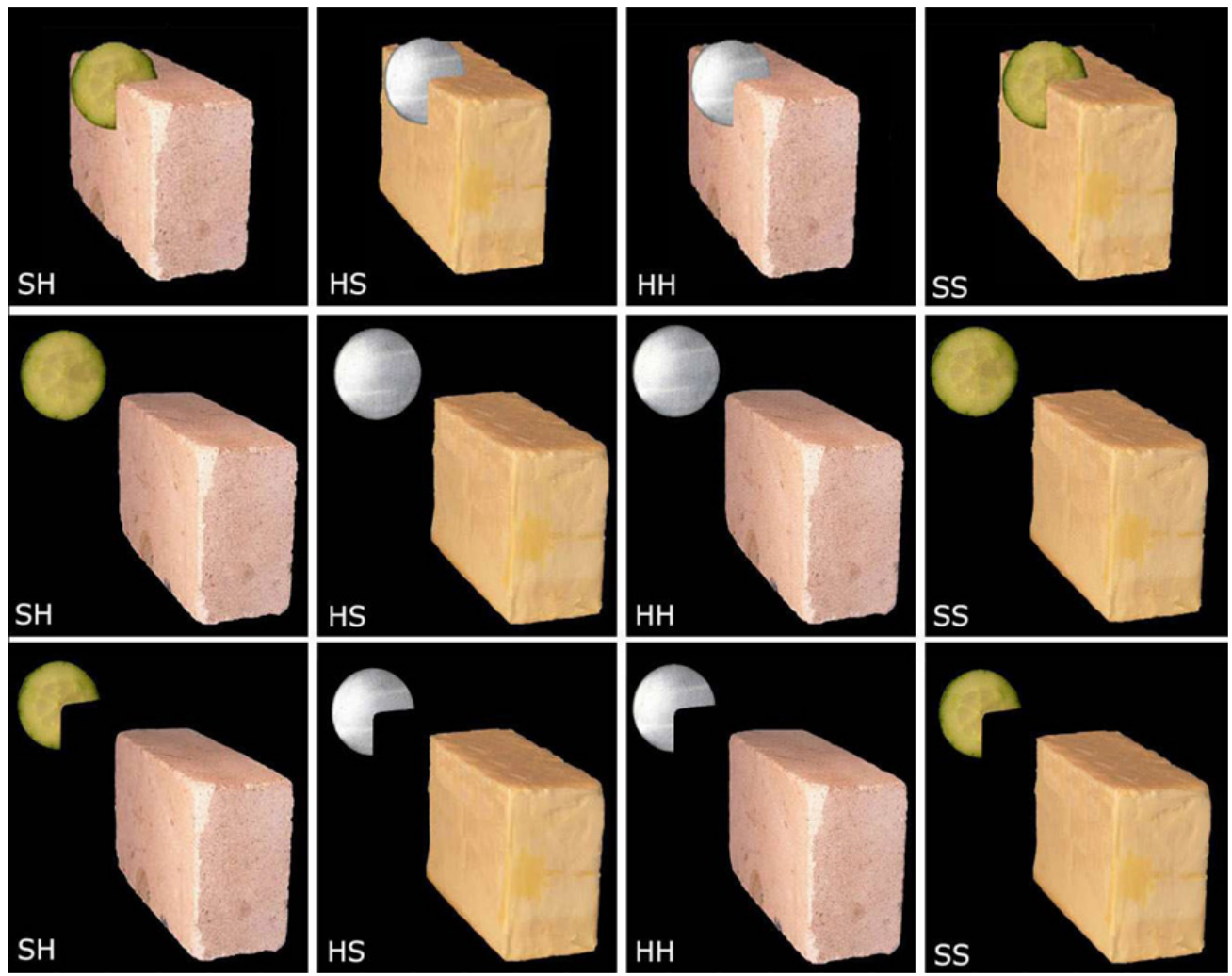

Figure 4. [In colour online, see http://dx.doi.org/10.1068/p6018]. Penetration, full-circle, and notchedcircle primes for all four prime material combinations: soft - hard (SH), hard - soft (HS), hard - hard $(\mathrm{HH})$, and soft-soft (SS).

In line with an influence of overall object interpretation on amodal completion, we expect that object semantics can also be influential during the amodal-completion process. Take, for example, the naturalistic stimuli of figure 4 . There, the circular slice is either a cucumber (soft) or a metal disc (hard). Similarly, the block can either be a brick (hard) or a block of butter (soft). These variations of material hardness might have different effects on amodal completion. It may be that the relative hardness of the block and the slice affects amodal completion, considering that the combination of a hard slice and a soft block is most likely to support amodal completion of the semicircular slice. Alternatively, amodal completion may depend largely on the hardness of the semicircular slice (eg only hard slices are perceived to penetrate the block) or may arise mainly from the hardness of the block (eg only soft blocks yield amodal completion of the slice). We conducted two experiments in which we tested the influence of the knowledge of the material hardness of objects on perceived object penetrations, using naturalistic stimuli and probing different prime durations: $300 \mathrm{~ms}$ in experiment 1; and $150 \mathrm{~ms}$ and $500 \mathrm{~ms}$ in experiment 2.

\section{Experiment 1}

\subsection{Method}

2.1.1 Participants. Twenty-four students participated in the experiment and received course credits. All subjects were naive with respect to the experimental hypotheses and had normal or corrected-to-normal vision. 
2.1.2 Stimuli. The primes were colour images of natural objects, displayed against a black background (figure 4). Each prime consisted of a (semi)circular slice on the left and a block on the right. The slice was either a (soft) cucumber slice or a (hard) metal disc; the block was either a typical block of (soft) Dutch butter or a (hard) brick. With these natural objects, four combinations (slice-block) were made based on the hardness of the material (referred to as prime material): soft - hard (SH), hard - soft (HS), hard hard $(\mathrm{HH})$, and soft-soft (SS). For each, there were three prime types: penetration primes (maximal horizontal extent $3.0 \mathrm{deg}$, maximal vertical extent $3.5 \mathrm{deg}$ ) and, to test for basic priming effects, full-circle and notched-circle primes (maximal horizontal extent $3.4 \mathrm{deg}$, maximal vertical extent $3.5 \mathrm{deg}$ ). The relevance of basic priming effects is further explained in section 2.2. When displaying the prime, the centre of the circle or notched circle was always positioned where the fixation cross had been previously. In sum, there were twelve different primes and a no-prime (np). The no-prime consisted of a rotated cross (maximal horizontal extent $0.3 \mathrm{deg}$, maximal vertical extent $0.3 \mathrm{deg}$ ).

Test-pairs always consisted of two (semi)circular slices that were either matching (eg two full cucumber slices) or non-matching (eg a full and a notched-circle cucumber slice). The objects within a test-pair differed only in shape, not in material. However, note that in the design there were trials in which the circular slice of the prime and the circular shapes of the test-pair differed in material (material-incongruent trials). These trials were, in fact, filler trials, added to have a fully balanced design in which all possible prime test-pair combinations occur.

The two objects (maximal horizontal extent $5.0 \mathrm{deg}$, maximal vertical extent $1.0 \mathrm{deg}$ ) appeared on both sides of, and equidistant from where the fixation cross had been. To avoid the impression of two (semi)circular objects popping out from either side of the central (semi)circular shape of the prime, disturbing priming (eg Sekuler and Palmer 1992), the block was also present at the bottom of the test-pair screen.

2.1.3 Procedure. The experiment was run on a PC with a $60 \mathrm{~Hz}$ refresh rate colour monitor and a display area of $1024 \times 768$ pixels. The experiment was presented with SuperLab Pro version 2.0 (Cedrus Corp. 1999). Subjects were seated, with their heads resting on a chin-rest, approximately $150 \mathrm{~cm}$ from the screen in a dimly lit room. They were instructed to indicate, as quickly as possible, while minimising errors, whether the two objects in the test-pair were identical or not, by pressing one of two buttons, corresponding to 'same' or 'different'. Half of the subjects pressed the 'same' button with their dominant hand, half with their non-dominant hand. Before the experiment started, subjects performed a small practice set and were informed explicitly about the different objects that were displayed (ie brick, butter, cucumber, metal disc). Participants were instructed to say focused on the area of the fixation point during all trials.

The prime duration was set at $300 \mathrm{~ms}$, since Sekuler and Palmer (1992) found maximal priming effects to test-pairs of completed prime-shapes to range between 200 and $400 \mathrm{~ms}$ of prime duration. Furthermore, Zago et al (2005) reported that an optimal visual priming effect is obtained after approximately $250 \mathrm{~ms}$. Additionally, an EEG study on visual object recognition indicated a recognition-related component arising between 150 and $300 \mathrm{~ms}$ (Johnson and Olshausen 2003). Each trial comprised the following sequence: a fixation cross ('+' for $250 \mathrm{~ms})$, a blank screen $(250 \mathrm{~ms})$, one of the primes (300 ms), an ISI (17 ms) and one of the test-pairs (until a response was given).

The experimental setup had four independent variables: 4 prime materials ( $\mathrm{SH}, \mathrm{HS}$, $\mathrm{HH}, \mathrm{SS}$ ) and 3 prime types (penetration, full circle, notched circle), 2 test-pair shapes (full circle, notched circle), and 2 test-pair materials (soft, hard), and test pairs were either matching or non-matching. Including the no-prime trials for all 16 test-pairs, the total amounted to 112 trials. These were randomised for each subject and repeated 
four times. At any time during the session, participants could take a break by pressing the pause button in-between trials. In practice, maximally two breaks were taken. A short feedback message was displayed after each incorrect response.

\subsection{Results}

Analyses were performed on correct responses to matching test-pairs (eg Beller 1971) that shared material hardness with the semicircular object of the prime (materialcongruent trials). Note that, although material-incongruent trials were included to have a fully balanced design, these trials are difficult to interpret in terms of amodal completion processes, as both shape and material properties can change within a prime test-pair combination. Errors were removed from the data set, as well as RTs deviating more than $3 \mathrm{SD}$ from the mean, leaving $95.2 \%$ of correct answers. For three subjects, one missing value was replaced by condition means. Mean priming effects were calculated by subtracting the mean RT to a test-pair in a specific prime condition from the same test-pair's mean RT in the corresponding no-prime condition: $\mathrm{PE}(\mathrm{TP} \mid \mathrm{P})=\mathrm{RT}(\mathrm{TP} \mid \mathrm{NP})-\mathrm{RT}(\mathrm{TP} \mid \mathrm{P})$, where $\mathrm{PE}$ is priming effect, TP is any test-pair, $\mathrm{P}$ is any prime, and NP is no-prime. The priming effects are plotted in figure 5 . We conducted repeated-measures ANOVAs separately for the basic prime conditions and for the penetration prime conditions.

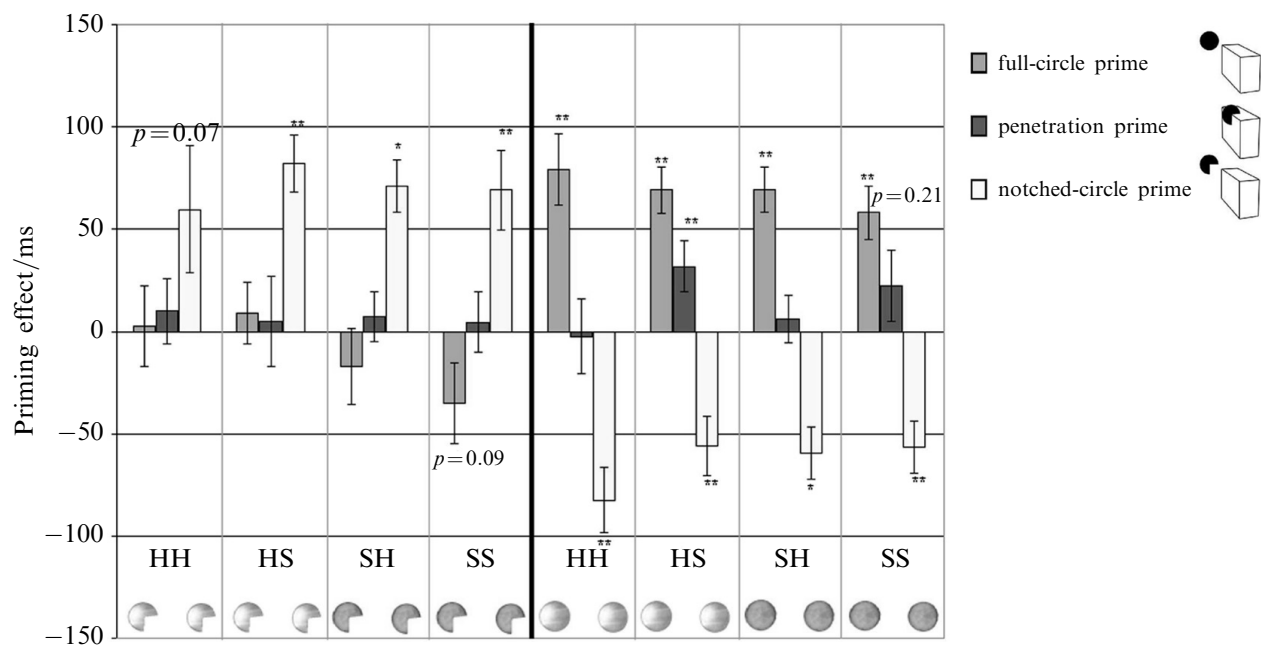

Figure 5. Priming effects of different prime types for the different prime materials [soft - hard (SH), hard - soft (HS), hard - hard (HH), and soft - soft (SS)] on notched-circle and full-circle test-pairs. Prime duration $=300 \mathrm{~ms}$. $* p<0.05, * * p<0.01$.

2.2.1 Basic prime conditions. The basic priming effects form an essential frame of reference when interpreting the effects of the penetration primes and are shape-congruent: full-circle primes should reduce RTs to full-circle test-pairs, and notched-circle primes should reduce RTs to notched-circle test-pairs. A repeated-measures ANOVA with 'prime material' (SH, HS, HH, SS), 'prime shape' (full circle, notched circle), and 'test-pair shape' (full circle, notched circle) was performed. There were main effects of 'prime shape' $\left(F_{1,23}=11.25, \mathrm{MSE}=5721.16, p<0.01\right)$ and 'test-pair shape' $\left(F_{1,23}=6.46\right.$, $\mathrm{MSE}=11829.90, p<0.05)$. As can be seen in figure 5, which also depicts the priming effects concerned in the analysis, these two main effects were caused by a series of significant negative priming effects by the mosaic prime on full-circle test-pairs. Owing to these negative effects, the overall priming effect of full-circle primes (28.8 ms) was greater than that of mosaic prime-shapes $(2.9 \mathrm{~ms})$. Accordingly, the overall priming effect for mosaic test-pairs $(30.0 \mathrm{~ms})$ was higher than that for full test-pairs $(1.8 \mathrm{~ms})$. 
There was also a significant interaction of 'prime material' and 'prime shape' $\left(F_{3,21}=3.21\right.$, $\mathrm{MSE}=2890.32, p<0.05)$. The interaction of 'prime material' and 'prime shape' is based on averages of the effects of one prime shape (eg full circle) on both full and notched circles. These averages are uninformative about shape-congruent primes and test-pairs (basic priming effects), neither adding to nor toning down the reliability of the basic priming effects.

Finally, there was a significant interaction of 'prime shape' and 'test-pair shape' $\left(F_{1,23}=178.90, \mathrm{MSE}=6095.67, p<0.01\right)$, which is rooted in the shape-congruent priming effects. $t$-Tests on paired-samples confirmed that, except for the marginally significant effect of the notched-circle prime in the HH condition $\left(t_{23}=1.93, p=0.07\right)$, all basic priming effects were significant. As can be seen in figure 5, all basic priming effects are shape-congruent.

2.2.2 Penetration prime conditions. The experimental factors were reduced to 'prime material' (SH, HS, HH, SS) and 'test-pair shape' (full circle, notched circle). Successively testing the three possible influences on completion proposed earlier, three ANOVAs were performed with different data pools. Testing for a differentiating effect on testpair shape caused by the relative hardness of the block and the slice, an ANOVA was run with the factors 'prime material' ( $\mathrm{SH}, \mathrm{HS}, \mathrm{HH}, \mathrm{SS}$ ) and 'test-pair shape' (full circle, notched circle). The interaction was not significant $\left(F_{3,21}=0.82, \mathrm{MSE}=4132.58\right.$, $p=0.48$ ). Figure 5 already reveals that the hardness of the circular slice alone (soft versus hard) did not differentially affect the completion of the slice either. This was confirmed by an ANOVA with the factors 'slice hardness' (soft, hard) and 'test-pair shape' (full circle, notched circle), that revealed no significant effect $\left(F_{1,23}=0.01\right.$, MSE $=3946.11$, $p=0.91)$. However, the hardness of the block did have an effect. An ANOVA comprising the factors 'block hardness' (soft, hard) and 'test-pair shape' (full circle, notched circle) revealed a significant interaction $\left(F_{1,23}=4.15, \mathrm{MSE}=2222.95, p<0.05\right)$. Paired-sample $t$-tests for the penetration prime conditions with soft blocks and their corresponding no-prime conditions revealed a significant priming effect for the HS-prime on full-circle test-pairs $\left(t_{23}=3.64, p<0.01\right)$ and a trend in the same direction for the SS-prime $\left(t_{23}=1.61, p=0.21\right)$.

\subsection{Discussion}

First, and of particular importance to the studies of naturalistic images, the basic priming effects proved reliable. This demonstrates that with more veridical stimuli the primed matching paradigm is usable.

In the penetration prime conditions, amodal completion of the semicircular slice was not affected by its relative hardness with respect to the block, nor by its own hardness. However, the significant interaction of the hardness of the block and the subsequent testpair shape indicates that the perceived material properties of the block do affect amodal completion of the adjoined slice. Specifically, a soft block yields amodal completion of the adjoined slice, whereas a hard block appears to leave the shape interpretation unresolved (as there are no priming effects on either test-pair shape). Indeed, the HS penetration condition revealed a significant priming effect on full-circle test-pairs. There was also a positive effect on full-circle test-pairs but it was not significant. Possibly, this is due to the fact that the overall material constitution of the SS penetration prime is more ambiguous, for it is debatable whether a soft block can be penetrated by a soft slice.

In the second experiment, set up similarly to experiment 1 , different prime durations were chosen (150 and $500 \mathrm{~ms}$ ) to explore whether the influence of object semantics, as measured after $300 \mathrm{~ms}$ of prime duration, also exists after shorter or longer prime exposures. In addition, extending the stimulus domain, some of the naturalistic objects were replaced by more typical alternatives: the soft butter with a block of typical Dutch cheese, the hard metal disc with a Euro coin. 


\section{Experiment 2}

\subsection{Method}

3.1.1 Participants. Twenty-eight students took part in the experiment and received course credits. All of them had normal or corrected-to-normal vision and were naive with respect to the research hypothesis.

3.1.2 Stimuli. The soft block was now of (typically soft) Dutch cheese and the hard slice was a 1-Euro coin. Figure 6 depicts the penetration primes (maximal horizontal extent $=2.5 \mathrm{deg}$, maximal vertical extent $=2.8 \mathrm{deg}$ ), full-circle primes, and notchedcircle primes (maximal horizontal extent $=3.4 \mathrm{deg}$, maximal vertical extent $=3.0 \mathrm{deg}$ ). Besides differing in shape, the non-matching test-pairs could also differ in their material properties.
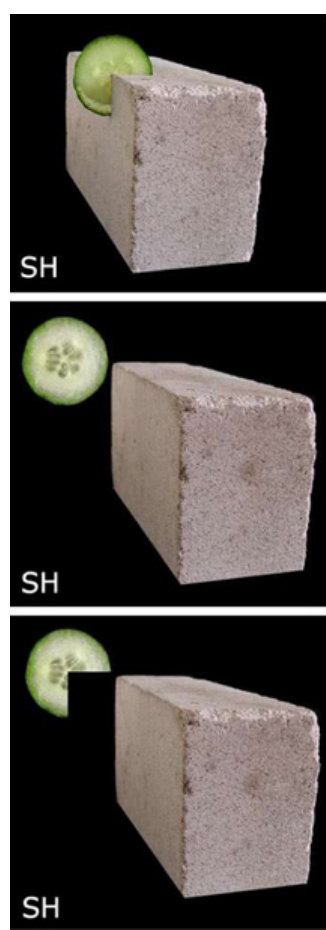
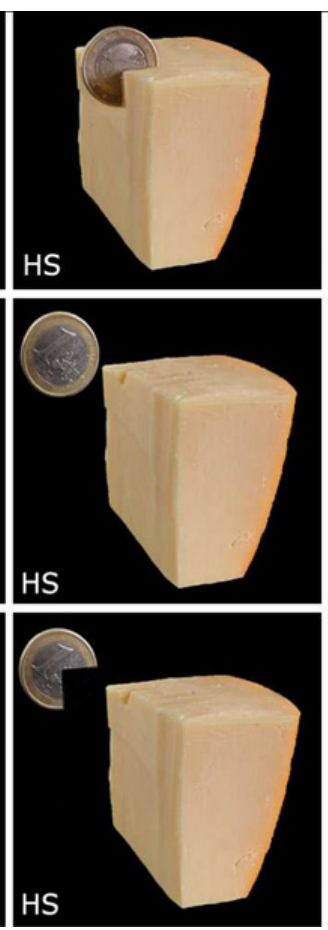
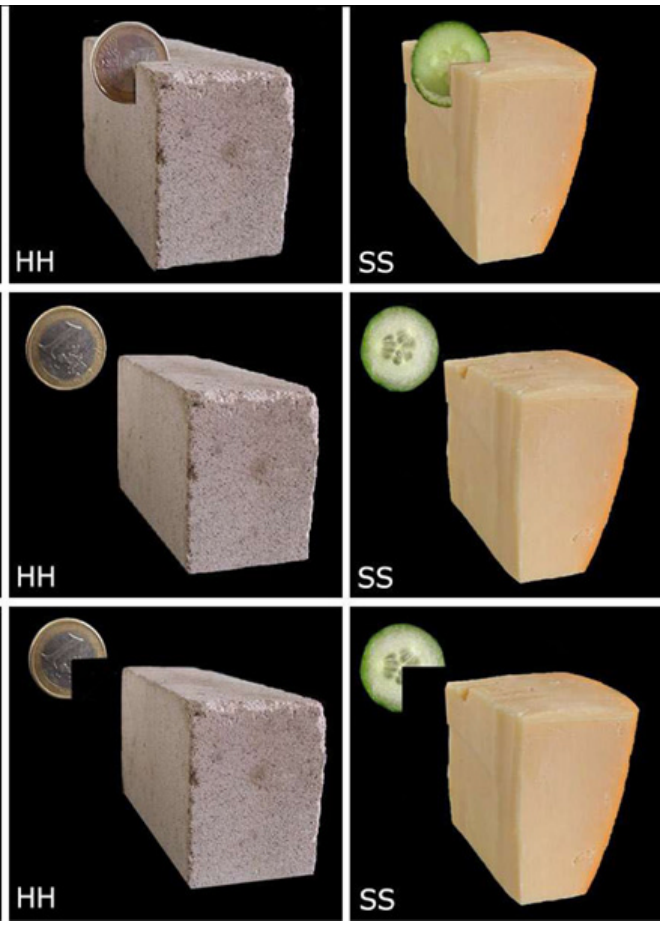

Figure 6. [In colour online.] The three prime types for the soft-hard (SH), hard-soft (HS), hard-hard (HH), and soft-soft (SS) conditions of experiment 2.

3.1.3 Procedure. The experimental setup now consisted of five independent variables: 'prime duration' (150 ms and $500 \mathrm{~ms}$ ), 'prime material' (SH, HS, HH, SS), 'prime shape' (full circle, notched circle, penetration), 'test-pair material' (hard, soft) and 'testpair shape' (full circle, notched circle). Additionally, test-pairs could be matching or non-matching, the latter stemming from differences in shape or material, resulting in three times as many non-matching test-pairs. Matching test-pair trials were repeated three times to counterbalance this asymmetry. The total number of trials, including the 48 no-prime conditions, was 624 .

\subsection{Results}

Again, we analysed RTs to correctly answered matching test-pairs that shared material hardness with the (semi)circular object of the prime. False responses were removed as well as RTs deviating more than 3 SDs from the mean (leaving 95.4\% of correct responses). One missing value of one participant was replaced by the condition mean. 
First, we present results of the basic prime conditions and after that the results of penetration prime conditions (first together, then separately for the $150 \mathrm{~ms}$ and $500 \mathrm{~ms}$ prime durations).

3.2.1 Basic prime conditions (150 $\mathrm{ms}$ and $500 \mathrm{~ms}$ conditions). As in experiment 1 , by analysing material-congruent trials only, a repeated-measures ANOVA was conducted, with the factors 'prime duration' (150 ms, $500 \mathrm{~ms})$, 'prime material' (SH, HS, HH, SS), 'prime shape' (full circle, notched circle), and 'test-pair shape' (full circle, notched circle). The mean priming effects of the $150 \mathrm{~ms}$ condition are plotted in figure 7, those of the $500 \mathrm{~ms}$ condition in figure 8.

As in experiment 1 , there was a significant main effect of 'prime shape' $\left(F_{1,27}=10.38\right.$, MSE $=2638.30, p<0.01$ ). This (again) stemmed from a higher average priming effect by full-circle primes $(36.0 \mathrm{~ms})$ than by notched-circle primes $(25.0 \mathrm{~ms})$, which was partially caused by a modest (non-significant) tendency of the notched-circle primes to yield

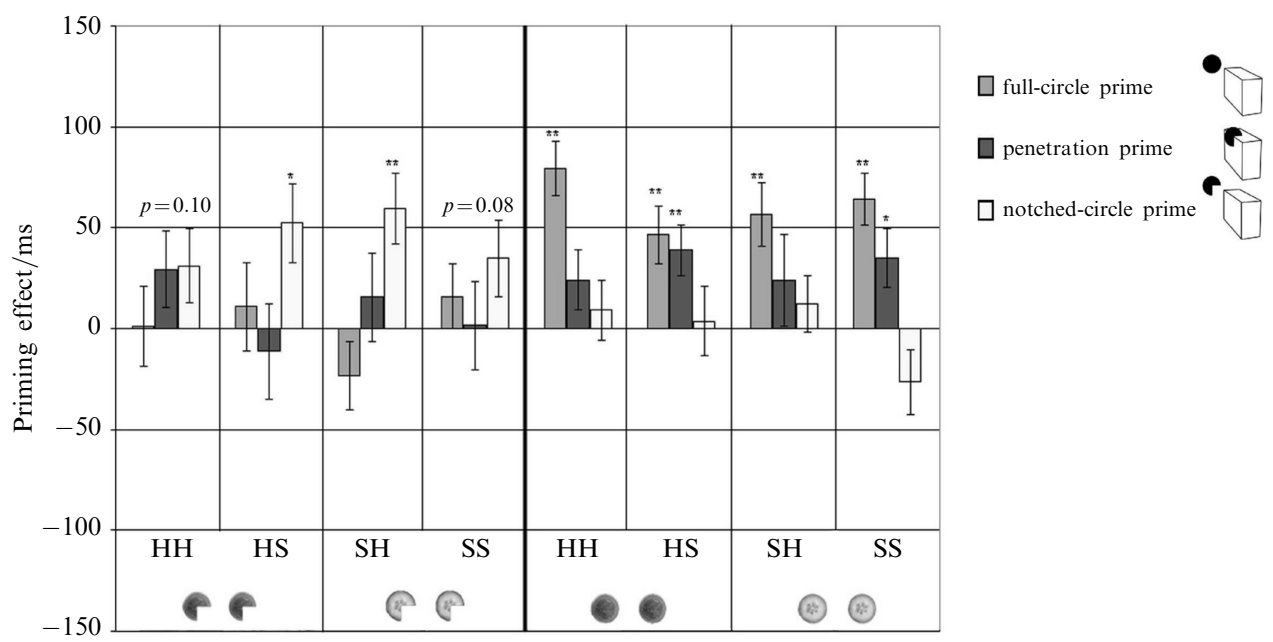

Figure 7. Priming effects of different prime types for the different prime materials [soft - hard (SH), hard-soft (HS), hard-hard (HH), and soft-soft (SS)] on notched-circle and full-circle test-pairs. Prime duration was $150 \mathrm{~ms} .{ }^{*} p<0.05, * * p<0.01$.

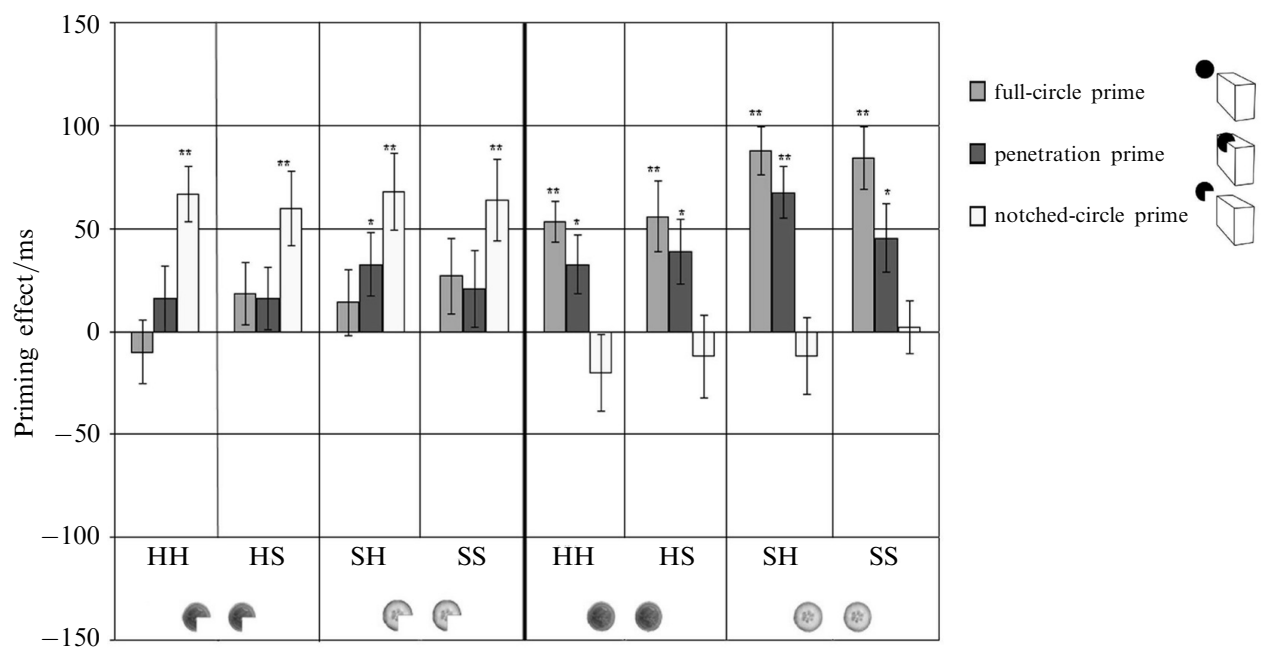

Figure 8. Priming effects of different prime types for the different prime materials [soft - hard (SH), hard-soft (HS), hard-hard (HH), and soft - soft (SS)] on notched-circle or full-circle test-pairs. Prime duration was $500 \mathrm{~ms} .{ }^{*} p<0.05, * * p<0.01$. 
negative priming effects on full-circle test-pairs in the $500 \mathrm{~ms}$ condition. In addition, in the $150 \mathrm{~ms}$ condition, two notched-circle prime conditions ( $\mathrm{HH}$ and SS on notchedcircle test-pairs) were only marginally significant $\left(t_{27}=1.69, p=0.10\right.$, and $t_{27}=1.85$, $p=0.08$, respectively), whereas all full-circle basic priming effects were significant. Further, and of main importance, there was a significant interaction of 'prime shape' and 'test-pair shape' $\left(F_{1,27}=80.62, \mathrm{MSE}=9889.41, p<0.01\right)$, which indicated that the different prime shapes differentially affected RTs to the two test-pair shapes. Indeed, paired-samples $t$-tests showed that all basic priming effects were significant, except for the $\mathrm{HH}$ and SS notched-circle prime in the $150 \mathrm{~ms}$ condition, as already mentioned. Finally, the ANOVA put out a three-way interaction between 'prime duration', 'prime material', and 'prime shape' $\left(F_{3,25}=3.41, \mathrm{MSE}=3240.90, p<0.05\right)$. This effect bears on the same averages as the two-way interaction of 'prime material' and 'prime shape', that was found and discussed in experiment 1 .

3.2.2 Penetration primes ( $150 \mathrm{~ms}$ and $500 \mathrm{~ms}$ conditions together). An ANOVA with the factors 'prime duration' (150 ms, $500 \mathrm{~ms}$ ), 'prime material' (SH, HS, HH, SS) and 'test-pair shape' (full circle, notched circle) revealed neither significant main effects nor interactions. There was a marginally significant main effect of 'test-pair shape' $\left(F_{1,27}=4.10, \mathrm{MSE}=14630.82, p=0.05\right)$. Averaged across all penetration conditions, priming effects were higher for full-circle $(38.3 \mathrm{~ms})$ than notched-circle test-pairs $(15.0 \mathrm{~ms})$. Figures 7 and 8 show that for both prime durations there are but small effects on notchedcircle test-pairs, and multiple significant priming effects on full-circle test-pairs.

3.2.3 Penetration primes (150 ms condition separately). Testing whether the relative hardness of the block and the slice has a differentiating effect on test-pair shape, an ANOVA was run with the factors 'prime material' ( $\mathrm{SH}, \mathrm{HS}, \mathrm{HH}, \mathrm{SS}$ ) and 'test-pair shape' (full circle, notched circle). No significant effects were found. To test whether the hardness of the circular slice differentially affected the completion of the slice, an ANOVA with the factors 'slice hardness' (soft, hard) and 'test-pair shape' (full circle, notched circle) was performed, revealing no significant results. However, as in experiment 1 , the block hardness did have an effect. An ANOVA was performed with the factors 'block hardness' (soft, hard) and 'test-pair shape' (full circle, notched circle), revealing a significant interaction between these two factors $\left(F_{1,27}=7.00, \mathrm{MSE}=1635.72, p<0.05\right)$. Zooming in, $t$-tests revealed significant priming effects for the HS-prime and SS-prime on full-circle test-pairs $\left(t_{27}=3.08, p<0.01\right.$, and $t_{27}=2.36, p<0.05$, respectively) and not on notched-circle test-pairs. In contrast, the $\mathrm{SH}$ and $\mathrm{HH}$ primes did not reduce RTs to those for full or notched-circle test-pairs.

3.2.4 Penetration primes (500 ms condition separately). The same ANOVAs were run for the $500 \mathrm{~ms}$ condition but provided no significant main effects or interactions. In each analysis, there was an identical marginally significant main effect of test-pair shape $\left(F_{1,27}=3.32, \mathrm{MSE}=5205.04, p=0.08\right)$. As can be understood from figure 8 , this effect is rooted in the series of positive priming effects on full-circle test-pairs (smallest positive effect: $t_{27}=2.28, p<0.05$ ), in contrast to only one significant effect, of the SH-prime, on notched-circle test-pairs $\left(t_{27}=2.10, p<0.05\right)$.

\subsection{Discussion}

The penetration primes in the $150 \mathrm{~ms}$ condition of experiment 2 followed the same priming pattern as in experiment 1 , again showing a significant differential priming effect consistent with block hardness. Penetration primes with soft blocks yielded priming effects on full-circle test-pairs but not on notched-circle test-pairs, whereas penetration primes with hard blocks yielded no priming effects.

In the $500 \mathrm{~ms}$ condition, all penetration primes facilitated RTs to full-circle testpairs. The differential priming effects due to block hardness, obtained in the $150 \mathrm{~ms}$ 
prime condition and in the $300 \mathrm{~ms}$ prime condition of experiment 1 , were no longer found. Possibly, the figural properties of the prime (eg good continuation, good form, favouring a full-circle interpretation) are more dominant towards the final stages of completion, disregarding familiarity with the material constitution of the prime that may suggest otherwise. However, in the $500 \mathrm{~ms}$ condition, there is a remarkable result in the SH penetration condition, which reveals a significant priming effect on both notched-circle and full-circle test-pairs. These effects seem related to the different possible interpretations of this particular prime. Its effect on full-circle test-pairs is similar to the priming effects of the other penetration primes and likely to be triggered by the figural aspects of the stimulus. Its priming effect on the notched-circle test-pairs, however, might be due to the specific material constitution of the SH penetration prime. More than the other penetration primes, the material constitution of the SH-prime (eg the top-left configuration in figure 6) favours a notched-circle interpretation of the slice. All in all, the priming effects on both test-pair shapes might reflect the ambiguity that is phenomenologically apparent in such configurations, demonstrating that amodal completion is not impregnable to the influence of object semantics.

As in experiment 1 , the primes in experiment 2 show effects on naturalistic object completion that, in one way or another, are consistent with the material properties of the objects involved. The fact that the hardness of the block (the occluder) and not the hardness of the slice (the potentially partly occluded object) affects completion poses an interesting asymmetry that we can only speculate on. One thought would be that the features of the block are more dominantly present in the display, simply because of its relative size.

\section{General discussion}

In the shorter prime-duration conditions $(150 \mathrm{~ms}$ and $300 \mathrm{~ms})$ the familiarity with the material hardness of the block (the occluder) strongly affected the perceived form of the adjoined notched slice. A soft block yielded a percept of a completed (hard or soft) circular slice, a hard block did not. In other words, amodal completion of the slice varies consistently with what one knows about the properties of the block. Importantly, this is apparent even with a prime duration as short as $150 \mathrm{~ms}$. If attributing our effects to object semantics, which we are inclined to, this information needs to be readily available. Next, we discuss whether such availability is plausible.

An early influence of material properties on amodal completion seems counterintuitive if we assume that knowledge about an object's material hardness is retrieved only after object recognition. In line with the four consecutive stages of visual processing as proposed by Marr (1982), researchers generally agree that 2-D retinal images work their way through stages of increasing complexity (eg from edges and lines to surfaces, and from surfaces to objects), until their information content reaches a categorical stage. At this processing stage, the functional properties of the object (eg material hardness) become available. If detected edges and lines are of the first features available in vision, one would expect a primacy of these figural aspects during amodal completion as well. Instead, we find consistent full-circle completions of the slice (as one would expect if completion had been based solely on figural aspects) only in the $500 \mathrm{~ms}$ conditionthe longest prime-duration condition.

Shape completion of simple geometrical occlusion configurations might very well run its course within early stages of vision just as it has been found by, for example, Sekuler and Palmer (1992). But in the case of more veridical stimuli early completion might be dominated by certain categorical information. That is, in early stages responses are merely based on a first-glance gist of the scene (eg stone cannot be penetrated, cheese can). We speculate here that with longer prime durations this first gist can then be either validated or overruled by a form-based amodal completion. 
Notice, however, that in the final impression some of the ambiguity may remain (that is, a banana penetrating a brick remains a curious state of affairs). As already mentioned, the priming effects we found in the $500 \mathrm{~ms} \mathrm{SH}$ condition on both mosaic and full-circle interpretations might reflect the perceptual duality that is phenomenologically present in the prime. This particular result can be reconciled with the lack of an effect of object familiarity found by Gerbino and Zabai (2003). After all, participants' decisions on Gerbino and Zabai's (2003) forced-choice task, take place well after $500 \mathrm{~ms}$. Clearly, Gerbino and Zabai's (2003) stimulus of joined naturalistic objects (comparable to both figure 3 and the top left of figure 4) carries a phenomenological dubiousness, which probably made it so attractive to investigate in the first place.

There is an important and interesting difference between Sekuler and Palmer's (1992) conclusions and our findings. This concerns the time-course of amodal completion. According to Sekuler and Palmer's two-stage model, amodal completion of pictorial, simple objects takes about $200 \mathrm{~ms}$ to reach its final (second) stage, consequently preempting the mosaic interpretation of the first stage. In contrast, according to our data, $150 \mathrm{~ms}$ is sufficient for visual completion of a naturalistic object, even taking the material properties of the occluder into account. Bruno et al (1997) have argued that the pictorial stimuli used by Sekuler and Palmer contain several ambiguities (eg binocular disparity, accommodation) that support a mosaic interpretation. When these are disambiguated, Bruno et al find completion effects even at $100 \mathrm{~ms}$ of stimulus presentation. Such a short completion time was also found by Rauschenberger et al (2004). One could argue that, although our stimuli are more complex (eg in terms of shading, colour, and perspective), they are perceptually also less ambiguous, yielding early shape interpretations of occluded objects.

In several studies of the time-course of object recognition early availability of categorical information is presumed. For instance, Johnson and Olshausen (2003) found an EEG component related to object recognition arising already around $150 \mathrm{~ms}$. Rapid serial visual presentation (RSVP) studies (eg Potter 1976) have shown that even at a speed of $133 \mathrm{~ms}$ per image, observers are able to detect cued picture or word categories. Similarly, Grill-Spector and Kanwisher (2005) demonstrated in a series of experiments that object detection (knowing that an object is present) and object categorisation (what kind of object it is) proceed equally fast and well within $100 \mathrm{~ms}$. Also, sceneperception studies (eg VanRullen 2007) demonstrate that an approximate $100 \mathrm{~ms}$ of stimulus presentation can be sufficient for basic category detection, or capturing the gist of a scene. VanRullen suggests that this fast scene interpretation results from a single, 'ultra-rapid', and inattentional feedforward sweep of neuronal activation, which is followed by visual awareness of details. A similar approach is outlined in the reverse hierarchy theory by Hochstein and Ahissar (2002), in which a single inattentional feedforward sweep is assumed to precede vision with scrutiny. To summarise, there are ample findings that suggest the availability of higher-order object information within the first few hundred milliseconds.

Thus far, models of amodal completion have been mostly based on the effects of figural, shape-based object properties (van Lier 1999; Tse 1999; Rubin 2001; de Wit and van Lier 2002). However, because these models relate amodal completion to the accurate processing of figural stimulus aspects alone, none of them captures the influence of object semantics that we measured. Still, as suggested by the results for the $500 \mathrm{~ms}$ condition of experiment 2, figural aspects of a stimulus may eventually dominate amodal completion. This confirms that the figural approach taken in these models was not an inappropriate reduction with regard to stimulus properties. Nevertheless, the present research also demonstrates that initial judgments and responses may very well rely on object semantics, such as knowledge of material properties, as well. 


\section{References}

Beller H, 1971 "Priming: Effects on advance information in matching" Journal of Experimental Psychology $87176-182$

Boselie F, Wouterlood D, 1989 "The minimum principle and visual pattern completion" Psychological Research $5193-101$

Bruno N, Bertamini M, Domini F, 1997 "Amodal completion of partly occluded surfaces: Is there a mosaic stage?" Journal of Experimental Psychology: Human Perception and Performance $\mathbf{2 3}$ $1412-1426$

Cedrus Corp., 1999 "SuperLab Pro, Experimental Lab Software, Version 2.0"

Gerbino W, Zabai C, 2003 "The joint" Acta Psychologica 114331 - 353

Grill-Spector K Kanwisher N, 2005 "Visual recognition: As soon as you know it is there, you know what it is" Psychological Science $16152-160$

Hochstein S, Ahissar M, 2002 "View from the top: hierarchies and reverse hierarchies in the visual system" Neuron $36791-804$

Johnson J S, Olshausen B A, 2003 "Timecourse of neural signatures of object recognition" Journal of Vision $3499-512$

Kanizsa G, 1979 Organization in Vision (New York: Praeger)

Kanizsa G, 1985 "Seeing and thinking" Acta Psychologica 59 23-33

Kellman P J, Shipley T F, 1991 "A theory of visual interpolation in object perception" Cognitive Psychology $23141-221$

Lier R van, 1999 "Investigating global effects in visual occlusion: from a partly occluded square to the back of a tree-trunk" Acta Psychologica 102 203-220

Lier R van, Helm P van der, Leeuwenberg E, 1995 "Competing global and local aspects of visual occlusion" Journal of Experimental Psychology: Human Perception and Performance 21571 - 583

Lier R van, Leeuwenberg E, Helm P van der, 1995 "Multiple completions primed by occlusion patterns" Perception $23883-903$

Lier R van, Wagemans J, 1999 "From images to objects: Global and local completions of selfoccluded parts" Journal of Experimental Psychology: Human Perception and Performance 25 $1721-1741$

Marr D, 1982 Vision: A Computational Investigation into the Human Representation and Processing of Visual Information (San Francisco, CA: W H Freeman)

Potter M C, 1976 "Short-term conceptual memory for pictures" Journal of Experimental Psychology: Human Learning and Memory $2509-522$

Rauschenberger R, Peterson M A, Mosca F, Bruno N, 2004 "Amodal completion in visual search: Preemption or context effects?" Psychological Science 15351 - 355

Rock I, 1983 The Logic of Perception (Cambridge, MA: MIT Press)

Rubin N, 2001 "The role of junctions in surface completion and contour matching" Perception $30339-366$

Sekuler A, 1994 "Local and global minima in visual completion: effects of symmetry and orientation" Perception 23 529-545

Sekuler A, Palmer S, 1992 "Perception of partly occluded objects: a microgenesis analysis" Journal of Experimental Psychology: General $12195-111$

Tse P U, 1998 "Illusory volumes from conformation" Perception 27 977-992

Tse P U, 1999 "Volume completion" Cognitive Psychology 39 37-68

Tse P U, 2002 "A contour propagation approach to surface filling-in and volume formation" Psychological Review 10991 - 115

VanRullen R, 2007 "The power of the feed-forward sweep" Advances in Cognitive Psychology 3167 - 176

Wit T C J de, Bauer M, Oostenveld R, Fries P, Lier R van, 2006 "Magnetic brain responses to global and local influences in visual completion" NeuroImage 32 1815-1825

Wit T C J de, Lier R van, 2002 "Global visual completion of quasi-regular shapes" Perception $31969-984$

Wit T C J de, Mol K, Lier R van, 2005 "Investigating metrical and structural aspects of visual completion: Priming versus searching" Visual Cognition $12409-428$

Wit T C J de, Vrins S, Dejonckheere P J N, Lier R van, 2008 "Form perception of partly occluded shapes in 4-month-old infants" Infancy 13 660-674

Zago L, Fenske M J, Aminoff E, Bar M, 2005 "The rise and fall of priming: How visual exposure shapes cortical representations of objects" Cerebral Cortex $151655-1665$ 


\section{PERTEPTION}

VOLUME 382009

www.perceptionweb.com

Conditions of use. This article may be downloaded from the Perception website for personal research by members of subscribing organisations. Authors are entitled to distribute their own article (in printed form or by e-mail) to up to 50 people. This PDF may not be placed on any website (or other online distribution system) without permission of the publisher. 